Research Article

\title{
Protection of Brass in HCl Solution by L-Cysteine and Cationic Surfactant
}

\author{
Milan B. Radovanovic (iD, Zaklina Z. Tasic, Marija B. Petrovic Mihajlovic, \\ and Milan M. Antonijevic
}

Technical Faculty Bor, University of Belgrade, VJ 12, P.O. Box 50, 19210 Bor, Serbia

Correspondence should be addressed to Milan B. Radovanovic; mradovanovic@tfbor.bg.ac.rs

Received 24 January 2018; Accepted 10 April 2018; Published 14 May 2018

Academic Editor: Fabrizio Pirri

Copyright (c) 2018 Milan B. Radovanovic et al. This is an open access article distributed under the Creative Commons Attribution License, which permits unrestricted use, distribution, and reproduction in any medium, provided the original work is properly cited.

\begin{abstract}
Electrochemical behavior of brass and inhibitory effect of L-cysteine in $\mathrm{HCl}$ solution are investigated using electrochemical techniques in addition to SEM-EDS analysis. Results show that inhibition efficiency depends on inhibitor concentration and immersion time of brass electrode in inhibitor solution. Electrochemical test results point to formation of $\mathrm{Cu}(\mathrm{I})$-cysteine complex on the brass surface in chloride solutions with addition of different amounts of cysteine. The formed complex has an important role in inhibition of the corrosion process in the examined media because $\mathrm{Cu}(\mathrm{I})$-cysteine significantly reduces dissolution of brass. Curves obtained in acidic solution in the presence of cysteine after pretreatment in sodium dodecyl sulfate (SDS) by cyclic measurements and potentiodynamic polarization measurements indicate intense inhibition of corrosion processes. Efficient inhibition of brass dissolution is result of formation of a stable protective layer on the brass surface after pretreatment of the electrode in SDS solution. The brass surface is modified in sodium dodecyl sulfate solution in order to increase cysteine adsorption on the electrode surface and to improve inhibition efficiency. Inhibition mechanism of cysteine includes adsorption on active sites on the electrode surface, which is confirmed by SEM-EDS analysis of brass. Adsorption of cysteine in hydrochloric acid solution obeys the Langmuir adsorption isotherm. Also, Gibbs free energy of adsorption has a value of $-31.5 \mathrm{~kJ} / \mathrm{mol}$ and indicates strong adsorption of cysteine on the electrode surface.
\end{abstract}

\section{Introduction}

Brass is widely used in various industries due to its stability in aggressive environments. The environment in which chloride ions are present is one of these [1,2], and corrosion processes in these environments are a major problem [3]. Also, it was proved that the dissolution of copper in chloride solutions depended largely on the concentration of chloride ions [4-8]. During the years of examinations of dissolution of metals and alloys in chloride solutions, different ways were revealed to slow down the oxidative processes in such media. One of the easiest and least expensive methods is application of organic inhibitors [9-12]. Nevertheless, most compounds that can be used as copper and brass corrosion inhibitors are toxic and nonbiodegradable and pose a threat to environmental protection. In recent years, much attention has been devoted to finding nontoxic and biodegradable inhibitors in terms of environmental protection. Moreover, amino acids are particularly singled out as potentially good "green inhibitors" in different aggressive media [13-18]. In addition to amino acids, compounds such as purine and adenine showed good results as nontoxic corrosion inhibitors in different aggressive media [19-23]. Moreover, nontoxic compounds which can be used as potent inhibitors should contain heteroatoms which have strong affinity to specific metals or a ring with delocalized electrons. Nevertheless, the presence of a ring with delocalized electrons in the inhibitor structure also enables and facilitates the adsorption of the examined compounds on the metal or alloy surface [24-28]. Cysteine (Cys) is a very interesting amino acid due to the amino group in its structure and -SH group that has strong affinity to copper (Figure 1).

Moreover, the existence of the -SH group makes cysteine a potentially good corrosion inhibitor [1]. In this paper, the 


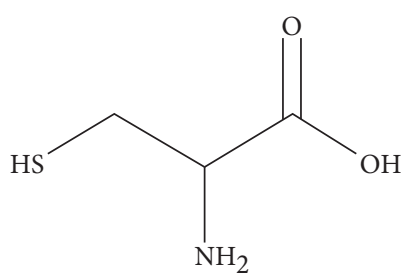

Figure 1: Cysteine structure.

inhibitory effect of cysteine on the brass oxidation in a hydrochloric acid solution was examined. During the work, primarily, the impact of cysteine concentrations on decrease of corrosion processes in the acidic chloride solutions was followed. Also, a suitable mechanism of corrosion inhibition was examined. Furthermore, pretreatment of the brass electrode in cysteine solution and in sodium dodecyl sulfate (SDS) solution for various periods of time was performed in order to obtain more complete insight into capability of cysteine as a corrosion inhibitor. Also, pretretment of brass electrode in these solutions was performed in order to find conditions for better adsorption of cysteine molecules on the electrode surface.

\section{Materials and Methods}

2.1. Electrochemical Measurements. Electrochemical measurements were performed using a potentiostat (Polarographic Analyzer PA2, Laboratorni Přistroje Prague, Czech Republic) that was directly connected to a computer via an $\mathrm{AD}$ card. The working electrode $(\mathrm{Cu} 37 \mathrm{Zn})$ was prepared by the method of casting upward; afterwards, it was sealed in a mixture based on methyl methacrylate. $\alpha+\beta$ brass was used for electrode preparation. Electrochemical measurements were performed in a three-electrode system where the working electrode was already described brass electrode, a saturated calomel electrode (SCE) was the reference electrode, and the platinum electrode was the auxiliary electrode. Prior to each measurement, the $\mathrm{Cu} 37 \mathrm{Zn}$ electrode was polished with alumina paste $(0.3 \mu \mathrm{m}$ $\mathrm{Al}_{2} \mathrm{O}_{3}$, Buehler USA) and then rinsed with distilled water and dried afterwards. Electrochemical methods applied during the experimental work were open circuit potential (OCP) measurements during $60 \mathrm{~min}$, linear potentiodynamic measurements, and cyclic voltammetry. Cyclic voltammetry was carried out from $-1.0 \mathrm{~V}$ versus SCE to $1.2 \mathrm{~V}$ versus SCE, whereas linear potentiodynamic measurements were performed from OCP to $-0.6 \mathrm{~V}$ versus SCE in the cathodic and to $0.25 \mathrm{~V}$ versus SCE in the anodic direction. Scan rates during linear potentiodynamic measurements and cycling voltammetry were $1 \mathrm{mV} / \mathrm{s}$ and $10 \mathrm{mV} / \mathrm{s}$, respectively.

The following compounds were used in preparation of the solution: hydrochloric acid $(\mathrm{HCl}$, Zorka Pharmacy Šbac), cysteine $\left(\mathrm{C}_{3} \mathrm{H}_{7} \mathrm{NO}_{2} \mathrm{~S}\right.$, Merck Germany), and sodium dodecyl sulfate $\left(\mathrm{NaC}_{12} \mathrm{H}_{25} \mathrm{SO}_{4}\right.$, J. T. Baker Germany) all of $\mathrm{p}$. a. purity. The stock solution of cysteine was $1 \cdot 10^{-2} \mathrm{M}$, while the set of working solutions $\left(5 \cdot 10^{-3}\right.$ to $\left.1 \cdot 10^{-4} \mathrm{M}\right)$ was obtained by diluting the stock solution. The electrochemical behavior of brass and action of cysteine in acidic chloride solutions were tested as follows: (i) Cyclic voltammetry was carried out in $0.05 \mathrm{M} \mathrm{HCl}$ in the presence of different concentrations of inhibitor $\left(1 \cdot 10^{-4}\right.$ to $\left.1 \cdot 10^{-2} \mathrm{M}\right)$.

(ii) Linear potentiodynamic measurements were performed after OCP measurements in $0.05 \mathrm{M} \mathrm{HCl}$ in the presence of various concentrations of inhibitor $\left(1 \cdot 10^{-4}\right.$ to $\left.1 \cdot 10^{-2} \mathrm{M}\right)$.

(iii) The electrode was immersed for a certain time (15, 30,60 , and $1140 \mathrm{~min}$ ) in $1 \cdot 10^{-2} \mathrm{M}$ cysteine and then rinsed with distilled water and transferred into the solution of hydrochloric acid in which cyclic voltammetry was carried out.

(iv) The electrode was immersed for various periods of time $(15,30,60$, and $1140 \mathrm{~min})$ in $1 \cdot 10^{-2} \mathrm{M}$ cysteine solution. Afterwards, the brass electrode was rinsed with distilled water and transferred into $0.05 \mathrm{M} \mathrm{HCl}$ solution in which, after measuring the open circuit potential, cathodic and anodic linear potentiodynamic measurements were performed.

(v) The brass electrode was immersed for a certain time, $15,30,60,1200$, and $4080 \mathrm{~min}$, in $1 \cdot 10^{-3} \mathrm{M}$ SDS and then rinsed with distilled water and transferred into $0.05 \mathrm{M} \mathrm{HCl}+1 \cdot 10^{-2} \mathrm{M}$ cysteine solution in which cyclic voltammetry was performed.

(vi) The working electrode was immersed for various periods of time $(15,30,60,1200$, and $4080 \mathrm{~min})$ in $1 \cdot 10^{-3} \mathrm{M}$ SDS solution and then rinsed with distilled water and transferred into $0.05 \mathrm{M} \mathrm{HCl}+1 \cdot 10^{-2} \mathrm{M}$ cysteine solution in which linear potentiodynamic measurements were carried out.

All measurements were done in naturally aerated solutions at ambient temperature. Also, all measurements were repeated at least three times, and presented curves represent the mean value of the measurements.

2.2. Surface Analysis. The SEM-EDS technique was used to examine the surface morphology of the brass after sample preparation without any treatment, after immersion of brass sample in $1 \cdot 10^{-2} \mathrm{M}$ cysteine solution, and after immersion of brass in $1 \cdot 10^{-3} \mathrm{M}$ SDS solution for 1140 and $4080 \mathrm{~min}$, respectively. The SEM/EDS experiments were performed on the Tescan VEGA 3 LM scanning electron microscope equipped with an Oxford EDS X-act Inca 350 system.

\section{Results and Discussion}

3.1. Open Circuit Potential Measurements. Open circuit potential measurements were done for $60 \mathrm{~min}$ in $0.05 \mathrm{M} \mathrm{HCl}$ solutions with and without the presence of various concentrations of cysteine. The obtained average values for OCP are shown in Figure 2(a).

A sharp shift of OCP values to the positive direction occurs after immersion of the brass electrode in chloride solution. Displacement of OCP values probably indicates that on the electrode surface occurs the formation of $\mathrm{CuCl}_{\text {ads }}^{-}$ intermediate according to equation $[1,29]$ : 


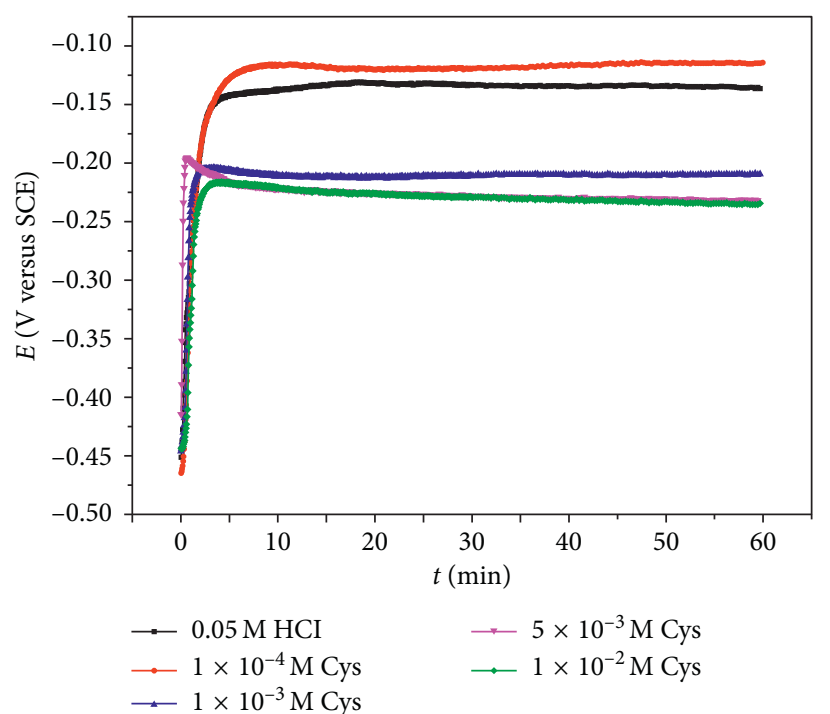

(a)

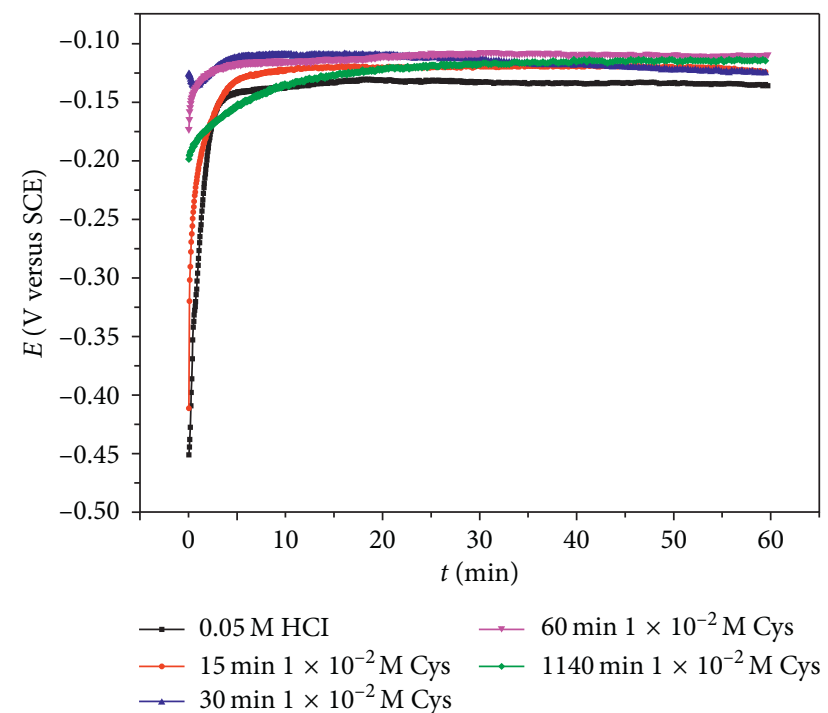

(b)

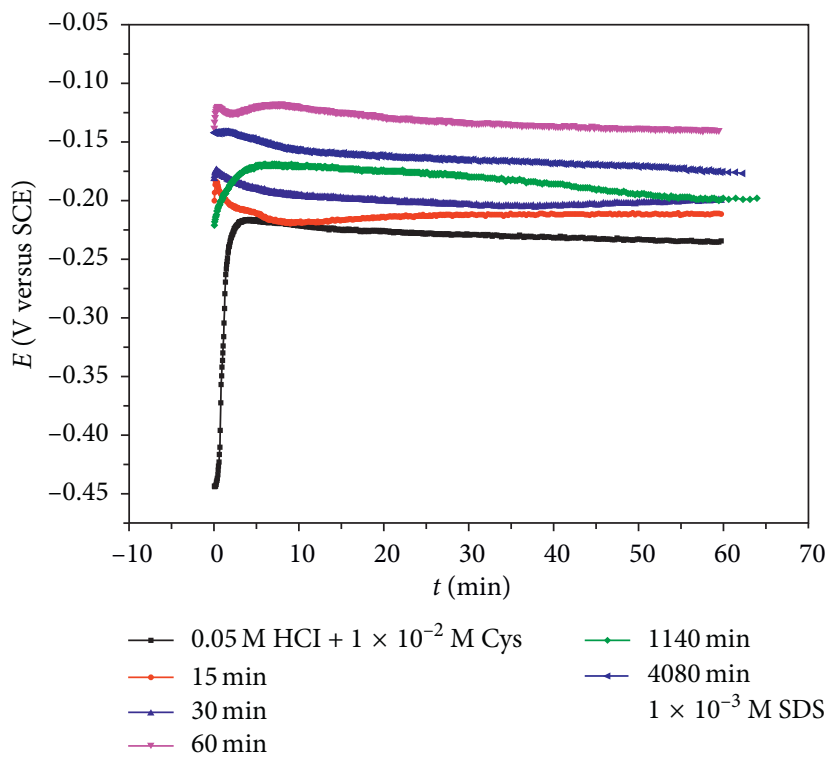

(c)

Figure 2: Open circuit potential of brass in $0.05 \mathrm{M} \mathrm{HCl}$ solution (a) without and with the addition of different concentrations of cysteine $\left(1 \cdot 10^{-4}\right.$ to $\left.1 \cdot 10^{-2} \mathrm{M}\right)$, (b) after immersion in $1 \cdot 10^{-2} \mathrm{M}$ cysteine solution for various periods of time $(15,30,60$, and $1140 \mathrm{~min})$, and (c) OCP of brass in $0.05 \mathrm{M} \mathrm{HCl}+1 \cdot 10^{-2} \mathrm{M}$ cysteine solution after immersion in $1 \cdot 10^{-3} \mathrm{M}$ SDS solution for various periods of time (15, 30, 60, 1200, and $4080 \mathrm{~min})$.

$$
\mathrm{Cu}+\mathrm{Cl}^{-}=\mathrm{CuCl}_{\mathrm{ads}}^{-}
$$

However, solubility of the formed intermediate is small, and precipitation of $\mathrm{CuCl}_{\text {ads }}^{-}$occurs on the electrode surface with the formation of $\mathrm{CuCl}$ layer. Also, OCP values obtained in hydrochloric solution in the presence of cysteine recorded upon reaching the steady state are generally negative relative to OCP values obtained in bare hydrochloric solution. Besides, displacement of steady state values of OCP is attributed to the adsorption of inhibitor molecules on the active sites on the electrode surface or to the formation of complex in the reaction between copper oxidation products and cysteine, which will be discussed later [30, 31]. Further, shift of OCP values to the negative direction in the presence of inhibitor indicates expected effect of cysteine as the cathodic type of inhibitor in acidic chloride solutions [31], and that will be also discussed later. Besides, it must be noted that adsorption of cysteine on the brass surface is greatly conditioned by the $\mathrm{pH}$ value of the solution. It is known that ionization of amino acids significantly depends on the $\mathrm{pH}$ value of the medium. Zwitter ion of cysteine is dominant species in aqueous solution in the $\mathrm{pH}$ range 1.91 to 8.16. Nonetheless, the protonated form of cysteine is the dominant form in solutions with $\mathrm{pH}$ values below 1.91. 


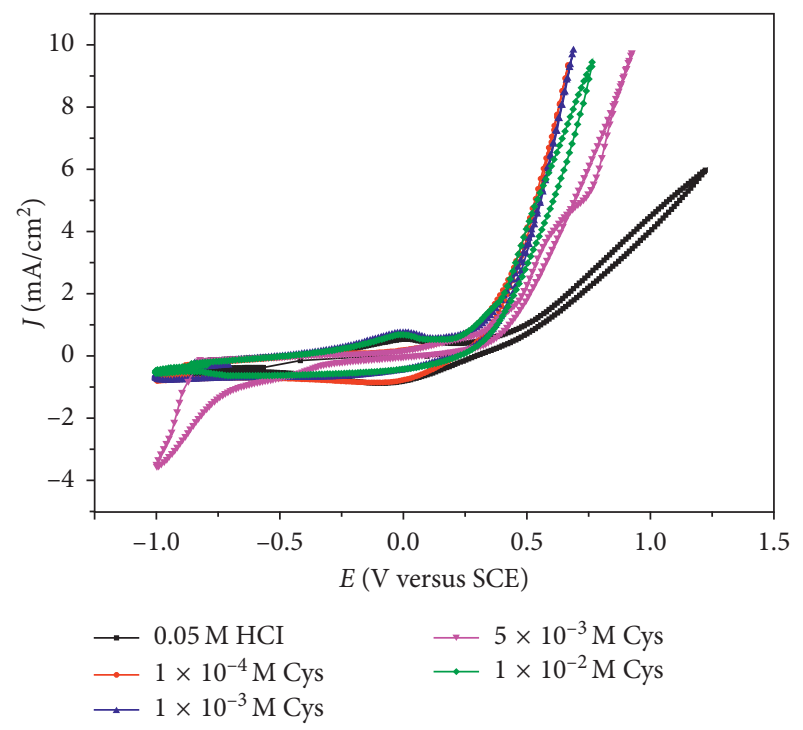

FIGURE 3: Cyclic voltammogram of brass recorded in $0.05 \mathrm{M} \mathrm{HCl}$ in the presence of various concentrations of cysteine (scan rate, $10 \mathrm{mV} / \mathrm{s})$.

Actually, the presence of the zwitterion and protonated form of cysteine in $0.05 \mathrm{M} \mathrm{HCl}$ contributes to the adsorption of the inhibitor on cathodic active sites on the brass surface [1].

$\mathrm{OCP}$ values obtained in $\mathrm{HCl}$ solution after pretreatment of the brass electrode in $1 \cdot 10^{-2} \mathrm{M}$ cysteine solution were not significantly changed in comparison with OCP values obtained in bare hydrochloride solution without pretreatment (Figure 2(b)). This indicates that cysteine acts like a mixedtype inhibitor, if there is any influence of cysteine on cathodic and anodic corrosion processes, and this will be discussed later in more detail. Nevertheless, cysteine is dominantly present in the form of the zwitterion in solution in which the pretreatment was carried out ( $\mathrm{pH}$ 5.96); that is, change in dominant species of the inhibitor occurs. Therefore the adsorption capacity of the cysteine decreases on cathodic active sites. This leads to the change of behavior of cysteine as a corrosion inhibitor of brass in hydrochloric solution.

OCP values obtained in $\mathrm{HCl}$ solution with the addition of $1 \cdot 10^{-2} \mathrm{M}$ cysteine after surface modification in sodium dodecyl sulfate solution are presented in Figure 2(c). The shift of OCP values up to $130 \mathrm{mV}$ in positive direction relative to the reference solution indicates that, after modification of electrode surface in SDS solution, adsorption of cysteine molecules occurs on anodic active sites. It can point to the fact that cysteine acts like an anodic-type inhibitor in the examined solution which will be discussed later. Moreover, upon determination of OCP values in $\mathrm{HCl}$ solution in the presence of cysteine and also after the brass surface modification in cysteine and SDS solutions, it can be noted that the state of the electrode surface had great influence on inhibitor adsorption and on the inhibition mechanism, which will be also discussed later.

\subsection{Influence of Cysteine Concentration}

3.2.1. Cyclic Voltammetry. Cyclic voltammogram of brass obtained in $\mathrm{HCl}$ solution with the addition of cysteine is shown in Figure 3.

Since the standard electrode potential for zinc is at more negative potentials compared to that for copper, the dissolution of zinc is expected to occur before copper dissolution by the following equation [32]:

$$
\mathrm{Zn}=\mathrm{Zn}^{2+}+2 \mathrm{e}^{-}
$$

Further, in chloride solutions also occurs reaction between zinc and $\mathrm{Cl}^{-}$ions according to the following equation [33]:

$$
\mathrm{Zn}+4 \mathrm{Cl}^{-}=\mathrm{ZnCl}_{4}^{2-}+2 \mathrm{e}^{-}
$$

Moreover, copper dissolution occurs in acidic solution which is shown by the following equations [4]:

$$
\begin{aligned}
\mathrm{Cu} & =\mathrm{Cu}^{+}+\mathrm{e}^{-} \\
\mathrm{Cu}^{+} & =\mathrm{Cu}^{2+}+\mathrm{e}^{-}
\end{aligned}
$$

Also, the anodic current peak observed on the CV curve (Figure 3) obtained in bare $\mathrm{HCl}$ solution points to the dissolution of $\mathrm{Cu}$ to $\mathrm{Cu}^{+}$. Thereafter, $\mathrm{CuCl}$ occurs in the reaction between $\mathrm{Cu}^{+}$and $\mathrm{Cl}^{-}$ions by the following equation:

$$
\mathrm{Cu}^{+}+\mathrm{Cl}^{-}=\mathrm{CuCl}
$$

Nevertheless, in the presence of atmospheric oxygen, oxidation of $\mathrm{Cu}^{+}$to $\mathrm{Cu}^{2+}$ occurs according to the following equation [34]:

$$
4 \mathrm{Cu}^{+}+\mathrm{O}_{2}+4 \mathrm{H}^{+}=4 \mathrm{Cu}^{2+}+2 \mathrm{H}_{2} \mathrm{O}
$$

Sudden increase of current density observed on CV curves obtained in $\mathrm{HCl}$ solution without and with the addition of the inhibitor $\left(1 \cdot 10^{-4}\right.$ to $\left.1 \cdot 10^{-2} \mathrm{M}\right)$ indicates weakening of adhesion of the adsorbed $\mathrm{CuCl}$ layer, its reaction with $\mathrm{Cl}^{-}$, and formation of soluble $\mathrm{CuCl}_{2}^{-}$complex [4]:

$$
\mathrm{CuCl}+\mathrm{Cl}^{-}=\mathrm{CuCl}_{2}^{-}
$$

Based on literature data, which refer to the conditions in the examined system, formed $\mathrm{CuCl}_{2}^{-}$complex can be dissolved into the bulk solution. Also, in acidic solution, oxidation of $\mathrm{CuCl}_{2}^{-}$to $\mathrm{Cu}^{2+}$ ions occurs according to the following equations [4]:

$$
\begin{aligned}
\mathrm{CuCl}_{2(\mathrm{ads})}^{-} & =\mathrm{CuCl}_{2 \text { (sol) }}^{-} \\
\mathrm{CuCl}_{2(\mathrm{ads})}^{-} & =\mathrm{Cu}^{2+}+2 \mathrm{Cl}^{-}+2 \mathrm{e}^{-}
\end{aligned}
$$

Moreover, it is reported that the dissolution of copper in acidic chloride solution is controlled by electrodissolution of copper and diffusion of $\mathrm{CuCl}_{2}^{-}$complex in the solution $[35,36]$.

In addition to the anodic processes, the dominant cathodic process in the examined solution is a process of oxygen reduction [37]:

$$
4 \mathrm{H}^{+}+\mathrm{O}_{2}+4 \mathrm{e}^{-}=2 \mathrm{H}_{2} \mathrm{O}
$$

The cathodic peak observed on CV curves in Figure 3 indicates the reduction of $\mathrm{CuCl}_{2}^{-}$complex and $\mathrm{CuCl}$ that are 


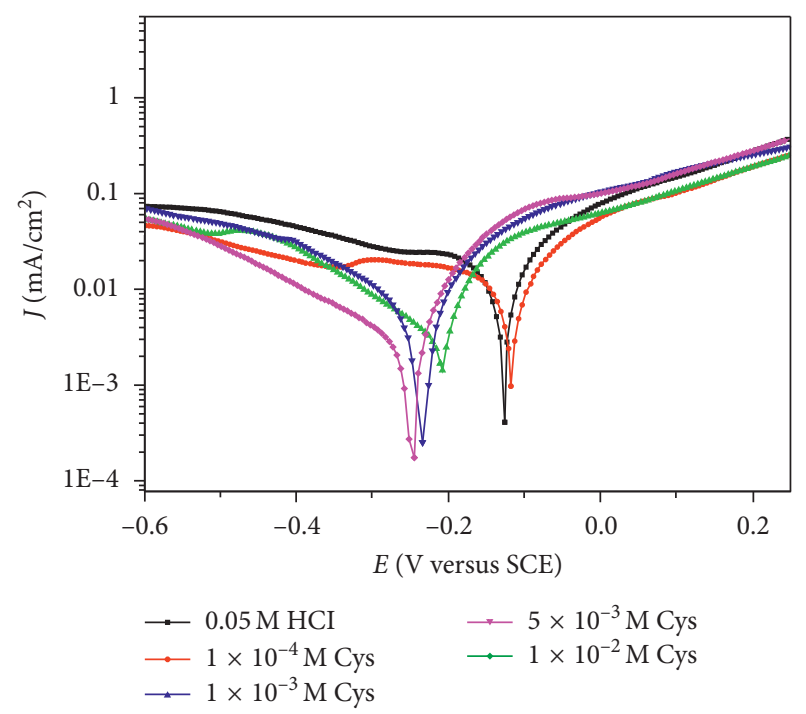

FIgUre 4: Polarization curves for brass in a $0.05 \mathrm{M} \mathrm{HCl}$ solution in the presence of various concentrations of cysteine (scan rate, $1 \mathrm{mV} / \mathrm{s}$ ).

formed on the brass surface [38]. Besides, the repassivation potential is noticed at $0.67 \mathrm{~V}$ versus SCE on the CV curve which was obtained in $0.05 \mathrm{M} \mathrm{HCl}$ solution with the addition of $5 \cdot 10^{-3} \mathrm{M}$ cysteine. Also, Ferreira et al. observed repassivation of copper in the chloride solution [39]. Further, the anodic current peak observed on CV curves obtained in $\mathrm{HCl}$ solution with an addition of cysteine indicates that oxidation of copper was achieved regardless of the presence of inhibitor. However, the cathodic current peak is not detected in $\mathrm{HCl}$ solution in the presence of cysteine except in the case when the cysteine was added at a concentration of $1 \cdot 10^{-4} \mathrm{M}$. Absence of the current peak indicates decrease of reduction processes of $\mathrm{CuCl}_{2}^{-}$and $\mathrm{CuCl}$, confirming the inhibitory effect of cysteine. Nevertheless, appearance of the cathodic current peak on the $\mathrm{CV}$ curve obtained in $\mathrm{HCl}$ solution in the presence of $1 \cdot 10^{-4} \mathrm{M}$ cysteine is a consequence of the insufficient amount of the inhibitor that is required to form a stable protective layer. Absence of reduction peaks in hydrochloric solution with an addition of cysteine in amount of $1 \cdot 10^{-3} \mathrm{M}$ up to $1 \cdot 10^{-2} \mathrm{M}$ is result of reaction between generated $\mathrm{Cu}^{+}$ions and cysteine molecules according to the following equation [40-42]:

$$
\mathrm{Cu}^{+}+\text {cysteine }=\mathrm{Cu}(\mathrm{I}) \text {-cysteine }+\mathrm{H}^{+}
$$

The reaction shown in (10) occurs primarily due to the fact that cysteine in its structure contains a thiol functional group. Accordingly, $\mathrm{Cu}^{+}$ions react with the thiol functional group and build a stable complex [11, 40,43]. However, the inhibitory effect of cysteine significantly decreases at higher potentials as can be seen on CV curves, which was caused by increase in the concentration of $\mathrm{Cu}^{2+}$ ions. Presence of $\mathrm{Cu}^{2+}$ leads to the cysteine oxidation according to the following equation [44]:

$$
6 \mathrm{RS}^{-}+2 \mathrm{Cu}^{2+}=\mathrm{RSSR}+2 \mathrm{RS}-\mathrm{Cu}-\mathrm{SR}
$$

Moreover, reduction of inhibitory activities of cysteine in acidic solutions is the result of electrooxidation of the inhibitor as shown in the following equation [45]:
TABLE 1: Corrosion parameters for brass in $0.05 \mathrm{M} \mathrm{HCl}$ solution with addition of various concentrations of cysteine.

\begin{tabular}{lcccc}
\hline $\begin{array}{l}C_{\text {inh }} \\
\left(\mathrm{mol} / \mathrm{dm}^{3}\right)\end{array}$ & $\begin{array}{c}E_{\text {corr }}(\mathrm{V}) \text { versus } \\
\text { SCE }\end{array}$ & $\begin{array}{c}j_{\text {corr }} \\
\left(\mu \mathrm{A} / \mathrm{cm}^{2}\right)\end{array}$ & $\begin{array}{c}b_{a} \\
(\mathrm{~V})\end{array}$ & $b_{c}(\mathrm{~V})$ \\
\hline $0.05 \mathrm{HCl}$ & -0.126 & 18 & 0.066 & -0.190 \\
$1 \cdot 10^{-4}$ Cys & -0.118 & 7 & 0.057 & -0.159 \\
$1 \cdot 10^{-3}$ Cys & -0.208 & 3 & 0.06 & -0.169 \\
$5 \cdot 10^{-3}$ Cys & -0.235 & 2.5 & 0.065 & -0.093 \\
$1 \cdot 10^{-2}$ Cys & -0.248 & 2 & 0.055 & -0.135 \\
\hline
\end{tabular}

TABLe 2: Polarization resistance and inhibition efficiency for brass in $0.05 \mathrm{M} \mathrm{HCl}$ solution with addition of various concentrations of cysteine.

\begin{tabular}{lcc}
\hline$C_{\text {inh }}\left(\mathrm{mol} / \mathrm{dm}^{3}\right)$ & $R_{\mathrm{p}}\left(\mathrm{k} \Omega \cdot \mathrm{cm}^{2}\right)$ & IE $(\%)$ \\
\hline $0.05 \mathrm{HCl}$ & 1.18 & - \\
$1 \cdot 10^{-4}$ Cys & 4.55 & 61.1 \\
$1 \cdot 10^{-3}$ Cys & 6.4 & 83.3 \\
$5 \cdot 10^{-3}$ Cys & 6.64 & 86.1 \\
$1 \cdot 10^{-2}$ Cys & 8.45 & 88.9 \\
\hline
\end{tabular}

$$
\mathrm{RSH}=\mathrm{RS}^{\cdot}+\mathrm{H}^{+}+\mathrm{e}^{-}
$$

The RS radical is further oxidized to the $\mathrm{RSO}_{2}$ species, which can be adsorbed on the electrode surface, or to the $\mathrm{RSO}_{3}^{-}$ ions which are the final product of cysteine oxidation [45].

3.2.2. Potentiodynamic Polarization Measurements. Besides the cyclic voltammetry, for a complete understanding of corrosion processes and mechanisms of action of cysteine, linear potentiodynamic measurements were performed.

Figure 4 shows the polarization curves of brass in $0.05 \mathrm{M}$ $\mathrm{HCl}$ in the presence of various concentrations of cysteine. Furthermore, polarization curves reveal that the addition of the inhibitor significantly diminishes cathodic corrosion processes. Further, corrosion potential shifts to more negative values in the presence of cysteine, indicating adsorption of the inhibitor on cathodic active sites on the electrode surface $[1,46]$. Also, displacement of corrosion potential values is mainly intensified with increasing concentration of the inhibitor.

In addition to adsorption of cysteine, a $\mathrm{Cu}(\mathrm{I})$-cysteine protective layer was formed on the electrode surface which acts by preventing the transfer of oxygen to the cathodic active sites on the electrode surface. Also, redeposition of copper ions occurs simultaneously with oxygen reduction in the examined system during cathodic corrosion processes [35]. Moreover, potentiodynamic polarization curves indicate that the inhibitory effect of cysteine is more pronounced in the vicinity of corrosion potential. However, intensification of corrosion processes occurs during polarization measurement as a result of decrease of the inhibition effect caused by protective film decomposition [40].

On the basis of polarization curves, electrochemical parameters of the brass oxidation were determined. Values of the corrosion potential $\left(E_{\text {corr }}\right)$, corrosion current density $\left(j_{\text {corr }}\right)$, and anodic and cathodic Tafel slopes $\left(b_{a}\right.$ and $\left.b_{c}\right)$ are presented in Table 1 . Values of polarization resistance $\left(R_{\mathrm{p}}\right)$ and inhibition efficiency (IE) are presented in Table 2. The 


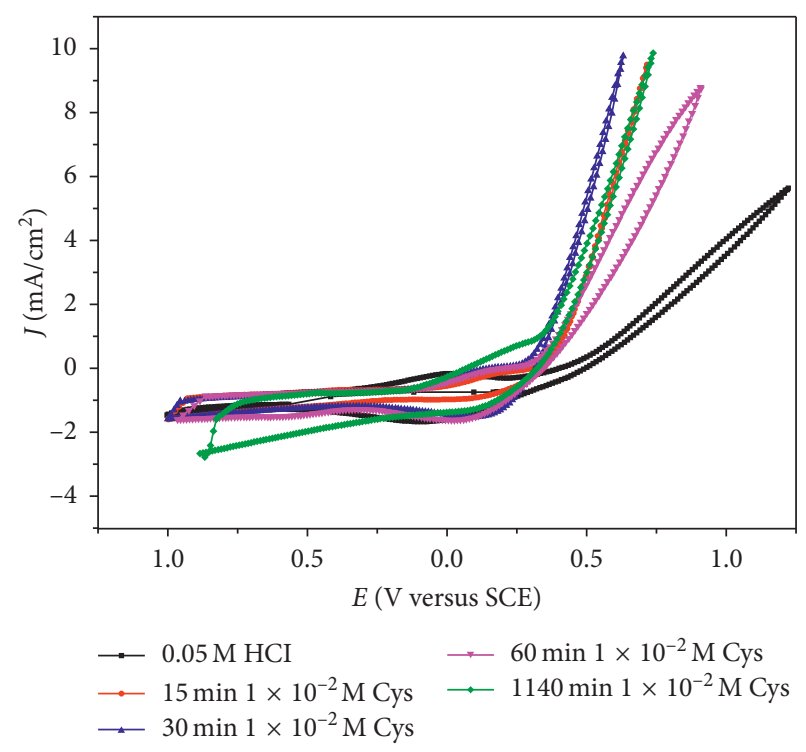

FIGURE 5: Cyclic voltammogram of brass in a $0.05 \mathrm{M} \mathrm{HCl}$ solution after immersion in $1 \cdot 10^{-2} \mathrm{M}$ cysteine solution for various periods of time $(15,30,60$, and $1140 \mathrm{~min}$; scan rate: $10 \mathrm{mV} / \mathrm{s})$.

inhibition efficiency is calculated according to the following equation:

$$
\mathrm{IE}=\left[\frac{\left(j_{\text {corr }}-j_{\text {inh }}\right)}{j_{\text {corr }}}\right] \cdot 100,
$$

where $j_{\text {corr }}$ and $j_{\text {inh }}$ represent corrosion current density in the absence and presence of the inhibitor.

According to $j_{\text {corr }}$ and IE values present in Tables 1 and 2, it can be seen that increase of cysteine concentration leads to current density decrease in examined solutions. However, values of anodic Tafel slopes are changed insignificantly in solution with the addition of cysteine, pointing to the fact that mechanisms of anodic processes remain unchanged with the addition of the inhibitor. Nonetheless, increase of cathodic Tafel slopes in the presence of the inhibitor is associated with cathodic current density decrease which indicates decrease of cathodic corrosion processes in test solution. Polarization resistance values were calculated according to the Stern-Geary equation $[47,48]$ :

$$
R_{\mathrm{p}}=\left[\frac{b_{a} \cdot b_{c}}{2.303}\left(b_{a}+b_{c}\right)\right] \cdot \frac{1}{j_{\text {corr }}} .
$$

Values of polarization resistance increase with increasing cysteine concentration, which can be seen in Table 2. Such change in $R_{p}$ values indicates adsorption of cysteine molecules on active sites on the brass surface, confirming the inhibitory action of cysteine [35, 49].

\subsection{Pretreatment of Brass Electrode in Cysteine Solution}

3.3.1. Cyclic Voltammetry. Cyclic voltammogram of brass obtained in $\mathrm{HCl}$ solution after immersion in water solution of $1 \cdot 10^{-2} \mathrm{M}$ cysteine for various periods of time $(15,30,60$, and $1140 \mathrm{~min}$ ) is shown in Figure 5.

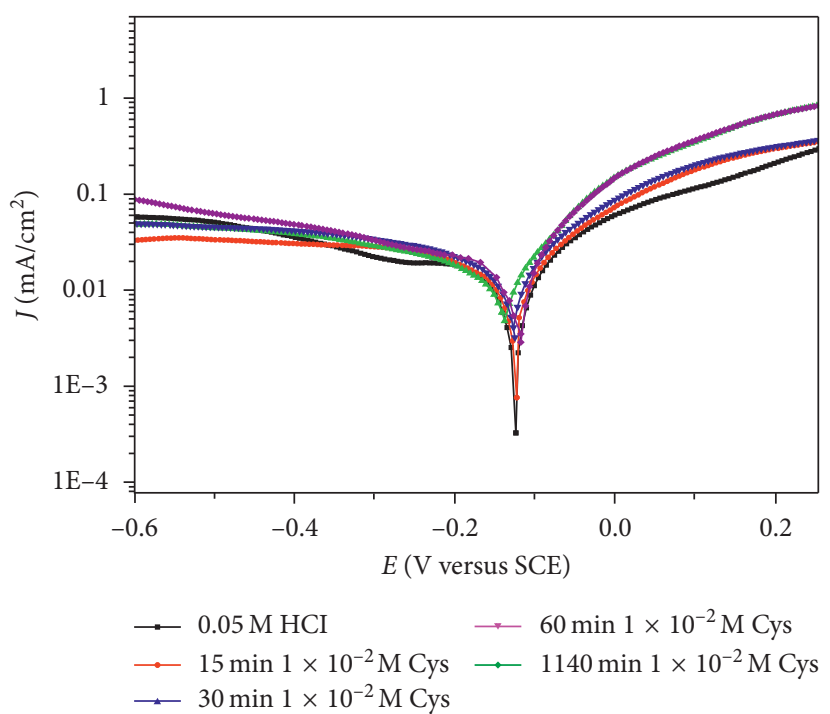

Figure 6: Polarization curves of brass in $0.05 \mathrm{M} \mathrm{HCl}$ solution after immersion in $1 \cdot 10^{-2} \mathrm{M}$ cysteine solution for various periods of time $(15,30,60$, and $1140 \mathrm{~min}$; scan rate, $1 \mathrm{mV} / \mathrm{s})$.

Based on CV curves, it can be seen that, after pretreatment of the electrode in inhibitor solution, solutions do not come to the significant change in electrochemical behavior of brass in $\mathrm{HCl}$ solution compared to cases when cysteine was present in $\mathrm{HCl}$ solution. Further, the anodic current peak is observed on $\mathrm{CV}$ curves obtained in $\mathrm{HCl}$ solution after pretreatment of the electrode in cysteine solutions as well in $\mathrm{HCl}$ solution with the addition of cysteine. Besides, the cathodic current peak is also detected in both cases whereby the current peak is more intense after pretreatment in inhibitor solution.

Intensification of the current peak indicates lower inhibitory effect of cysteine after immersion of electrode in inhibitor solution, which is probably the result of absence of chloride ions in the solution where pretreatment was performed.

Nonetheless, adsorption of cysteine on the brass surface was more enhanced in $\mathrm{HCl}$ solution with an addition of inhibitor in different amounts. Based on that, it can be said that adsorption process is favored by chloride ions present in the solution [50]. Moreover, chloride ions were adsorbed on metal surface easily, forming interconnecting bridges between the metal surface and positively charged molecules of the cysteine. Furthermore, formed interconnecting bridges promote adsorption of the inhibitor [51]. Moreover, chloride ions change the charge of the metal surface making the surface more affordable to protonated molecules of the inhibitor. During pretreatment of the brass electrode, chloride ions were not present in inhibitor solution, which means that charge change of the surface did not occur in inhibitor solution. Therefore, adsorption of cysteine molecules on the electrode surface is more difficult under such conditions.

3.3.2. Potentiodynamic Polarization Measurements. After cyclic voltammetry, linear potentiodynamic measurements were performed in $0.05 \mathrm{M} \mathrm{HCl}$ solution after pretreatment of the brass electrode in water solution of inhibitor for various periods of time $(15,30,60$, and $1140 \mathrm{~min})$. The obtained curves are shown in Figure 6. 


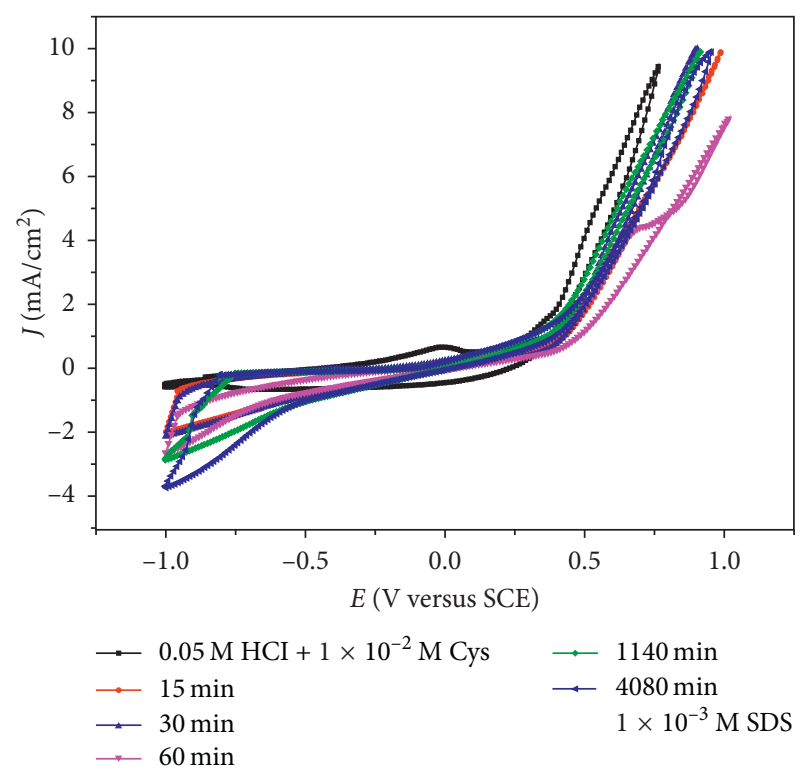

Figure 7: Cyclic voltammogram of brass in $0.05 \mathrm{M} \mathrm{HCl}+1 \cdot 10^{-2} \mathrm{M}$ cysteine solution after electrode immersion in $1 \cdot 10^{-3} \mathrm{M}$ SDS solution for various periods of time $(15,30,60,1200$, and $4080 \mathrm{~min}$; scan rate: $10 \mathrm{mV} / \mathrm{s}$ ).

Polarization curves of brass reveal that pretreatment of the brass electrode in cysteine solution has insignificant influence on decrease of corrosion processes in acidic chloride solution. Moreover, obtained polarization curves show that the cathodic current density does not change significantly in $\mathrm{HCl}$ solution. Besides, polarization curves obtained after electrode exposure to cysteine solution are approaching to the curve achieved in the $\mathrm{HCl}$ solution without prior immersion in the inhibitor solution. This also points to the reduction of the inhibitory effect of the protective layer formed on the electrode surface during pretreatment in the cysteine solution. Nonetheless, anodic polarization curves obtained in $\mathrm{HCl}$ indicate that electrode immersion in the inhibitor solution leads to the slight activation of the brass electrode in chloride solution and to the more pronounced dissolution of alloy in relation to the case when the cysteine was present in the $\mathrm{HCl}$ solution. Moreover, apparent brass dissolution is achievable, due to the fact that cysteine cannot be adsorbed on the anodic active sites on the electrode surface making these sites available to chloride ions. Besides, after pretreatment in cysteine solution for various periods of time, shapes of anodic and cathodic polarization curves achieved in $\mathrm{HCl}$ solution do not change in relation to the polarization curves obtained in hydrochloric solution without electrode pretreatment in the inhibitor solution. This indicates that mechanisms of anodic and cathodic corrosion processes did not change after electrode immersion in $1 \cdot 10^{-2} \mathrm{M}$ cysteine solution.

\subsection{Pretreatment of Brass Electrode in SDS Solution}

3.4.1. Cyclic Voltammetry. Figure 7 shows cyclic voltammogram of brass recorded in $0.05 \mathrm{M} \mathrm{HCl}+1 \cdot 10^{-2} \mathrm{M}$ cysteine

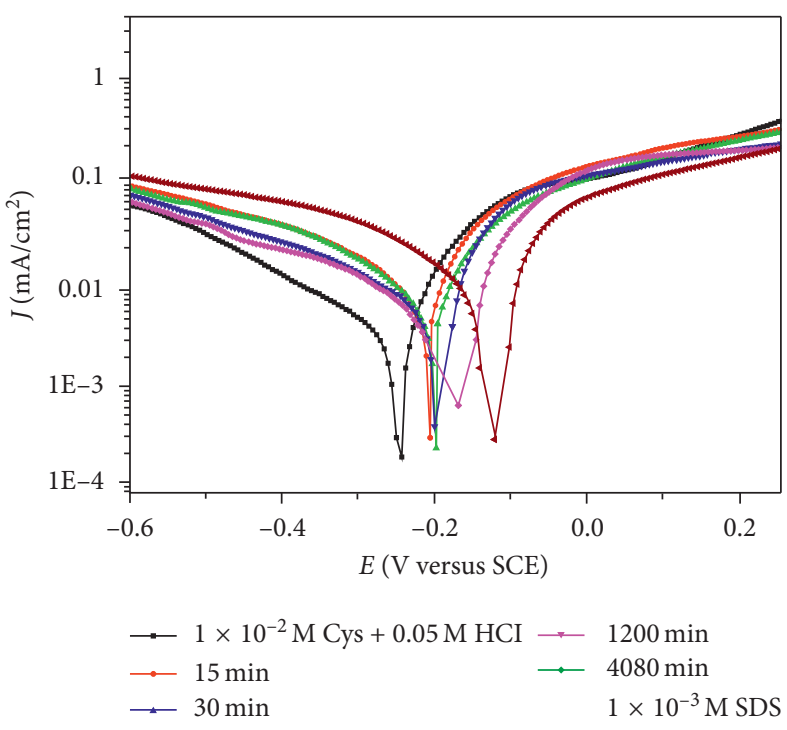

FIgURE 8: Polarization curves for brass in $0.05 \mathrm{M} \mathrm{HCl}+1 \cdot 10^{-2} \mathrm{M}$ cysteine solution after immersion in $1 \cdot 10^{-3} \mathrm{M}$ SDS solution for various periods of time $(15,30,60,1200$, and $4080 \mathrm{~min}$; scan rate, $1 \mathrm{mV} / \mathrm{s}$ ).

solution after immersion of the electrode for various periods of time $(15,30,60,1200$, and $4080 \mathrm{~min})$ in $1 \cdot 10^{-3} \mathrm{M}$ SDS solution.

The brass electrode was subjected to pretreatment in $1 \cdot 10^{-3} \mathrm{M}$ SDS solution for various periods of time and thereafter immersed in $\mathrm{HCl}$ solution with the addition of $1 \cdot 10^{-2} \mathrm{M}$ cysteine in order to improve protective effects of cysteine film that was formed on the brass surface. Moreover, CV curves obtained in acidic solution in the presence of cysteine indicate intense inhibition of corrosion processes after modification of the electrode in the SDS solution. Nonetheless, the surface of the electrode was more effectively covered with a stable protective layer after electrode immersion in SDS solution in relation to cases when the electrode was immersed in the cysteine solution or when electrode was exposed to $\mathrm{HCl}$ solution with the addition of cysteine without any pretreatment. During pretreatment in SDS solution, surfactant was adsorbed electrostatically on the brass surface through the ionized alkyl acid radical negative ions [52]. Applied concentration of SDS is near to the critical micelle concentrations (CMC) that causes the formation of micelles after prolonged exposure of the electrode in the sodium dodecyl sulfate solution [53, 54]. Furthermore, the negatively charged part of the SDS molecule facilitates the adsorption of the inhibitor on the electrode surface in acidic medium wherein cysteine is predominantly present in the cationic form. Moreover, adsorption density of SDS becomes higher with increasing immersion time of the brass electrode in SDS solution. This leads to interaction between tails of negative ions of SDS and formation of hemimicelles that leave the electrode surface [55]. The part of the electrode surface from which SDS ions went becomes available to cysteine molecules that adsorbed on the electrode and prevents contact between the electrode surface and aggressive ions. 
TABLE 3: Corrosion parameters for brass in acidic chloride solution after immersion in cysteine and SDS solutions for various periods of time.

\begin{tabular}{|c|c|c|c|c|c|}
\hline$C_{\text {inh }}\left(\mathrm{mol} / \mathrm{dm}^{3}\right)$ & $t(\min )$ & $E_{\text {corr }}(\mathrm{V})$ versus SCE & $j_{\text {corr }}\left(\mu \mathrm{A} / \mathrm{cm}^{2}\right)$ & $b_{a}(\mathrm{~V})$ & $b_{c}(\mathrm{~V})$ \\
\hline \multirow{4}{*}{$1 \cdot 10^{-2}$ Cys } & 15 & -0.129 & 9 & 0.079 & -0.099 \\
\hline & 30 & -0.140 & 8 & 0.072 & -0.155 \\
\hline & 60 & -0.127 & 7.5 & 0.067 & -0.262 \\
\hline & 1140 & -0.120 & 7 & 0.074 & -0.256 \\
\hline \multirow{5}{*}{$1 \cdot 10^{-3} \mathrm{SDS}$} & 15 & -0.213 & 3 & 0.048 & -0.075 \\
\hline & 30 & -0.201 & 2.3 & 0.05 & -0.0725 \\
\hline & 60 & -0.194 & 1.6 & 0.037 & -0.066 \\
\hline & 1200 & -0.173 & 1 & 0.028 & -0.049 \\
\hline & 4080 & -0.123 & 0.64 & 0.027 & -0.046 \\
\hline
\end{tabular}

TABLE 4: Polarization resistance and inhibition efficiency for brass in acidic chloride solution after immersion in cysteine and SDS solutions for various periods of time.

\begin{tabular}{lccr}
\hline$C_{\text {inh }}\left(\mathrm{mol} / \mathrm{dm}^{3}\right)$ & $t(\mathrm{~min})$ & $R_{\mathrm{p}}\left(\mathrm{k} \Omega \cdot \mathrm{cm}^{2}\right)$ & IE $(\%)$ \\
\hline \multirow{3}{*}{$1 \cdot 10^{-2}$ Cys } & 15 & 2.37 & 50.0 \\
& 30 & 2.4 & 55.5 \\
& 60 & 3.0 & 58.3 \\
& 1140 & 3.55 & 61.1 \\
$1 \cdot 10^{-3}$ SDS & 15 & 4.41 & 83.3 \\
& 30 & 5.57 & 87.2 \\
& 60 & 6.44 & 91.1 \\
& 1200 & 7.7 & 94.4 \\
\hline
\end{tabular}

3.4.2. Potentiodynamic Polarization Measurements. Cathodic and anodic potentiodynamic curves obtained in $0.05 \mathrm{M} \mathrm{HCl}$ $+1 \cdot 10^{-2} \mathrm{M}$ cysteine solution after pretreatment of the brass electrode for different periods of time $(15,30,60,1200$, and $4080 \mathrm{~min}$ ) in $1 \cdot 10^{-3} \mathrm{M}$ SDS solution are presented in Figure 8.

From presented potentiodynamic curves, it can be seen that the change in the brass behavior occurs as compared to the case when the electrode was immersed in cysteine solution. Moreover, polarization curves point to an increase of cathodic corrosion processes after pretreatment of electrode in SDS solution in relation to the previously described experimental conditions. Nonetheless, polarization curves obtained in acidic medium after pretreatment of the electrode in SDS solution indicate facilitated adsorption of cysteine on anodic active sites on the electrode surface. Accordingly, anodic polarization curves reveal that, after modification of the brass electrode in SDS solution, anodic corrosion processes were inhibited more efficiently, mainly in the vicinity of corrosion potential.

Electrochemical parameters of brass oxidation in the acidic chloride solution with immersion of the brass electrode in cysteine and SDS solutions were calculated on the basis of potentiodynamic curves (Figures 6 and 8 ), and obtained values for $E_{\text {corr }}, j_{\text {corr }}, b_{a}$, and $b_{c}$ are shown in Table 3.

Values of $R_{\mathrm{p}}$ and IE are presented in Table 4 in addition to the electrochemical parameters.

$E_{\text {corr }}$ values obtained in $\mathrm{HCl}$ solution after pretreatment in $1 \cdot 10^{-2} \mathrm{M}$ cysteine solution indicate a slight shift in the negative direction in regard to $E_{\text {corr }}$ values obtained in working solution without pretreatment in the inhibitor solution. Accordingly, shift of $E_{\text {corr }}$ values in correlation with the decrease of $j_{\text {corr }}$ values points to inhibitory action of cysteine on cathodic corrosion processes. Furthermore, cathodic Tafel slopes obtained from polarization curves achieved in $\mathrm{HCl}$ solution after electrode modification in cysteine solution recorded more intensive change in values in regard to $b_{c}$ values obtained in $\mathrm{HCl}$ solution without electrode modification. Accordingly, change of $b_{c}$ values indicates a decrease of cathodic corrosion processes in examined solutions. Nonetheless, slight deviation of values of anodic Tafel slopes obtained from polarization curves after pretreatment in cysteine solution, in comparison with values of anodic Tafel slopes obtained in $\mathrm{HCl}$ solution without any modification of electrode surface, indicates that mechanism of anodic corrosion processes remains unchanged.

$E_{\text {corr }}$ values, achieved in $0.05 \mathrm{M} \mathrm{HCl}+1 \cdot 10^{-2} \mathrm{M}$ cysteine solution after electrode modification in SDS solution, are shifted towards positive values indicating effortless adsorption of cysteine on anodic active sites on the modified surface of brass. Values of $j_{\text {corr }}$ obtained in acidic chloride solution decrease after pretreatment of brass in cysteine and SDS solutions. Moreover, decrease of $j_{\text {corr }}$ values is more pronounced after electrode modification in SDS solution pointing to more efficient inhibition of corrosion processes. Besides, the degree of inhibition efficiency increases with the increase of pretreatment duration in cysteine and SDS solutions. Moreover, IE values are much higher after electrode modification in SDS solution. Cathodic and anodic Tafel slope values obtained in acidic chloride solution after electrode modification in SDS solution indicate brass passivation due to the formation of protective film on the electrode surface $[9,56]$. Also, polarization resistance values 

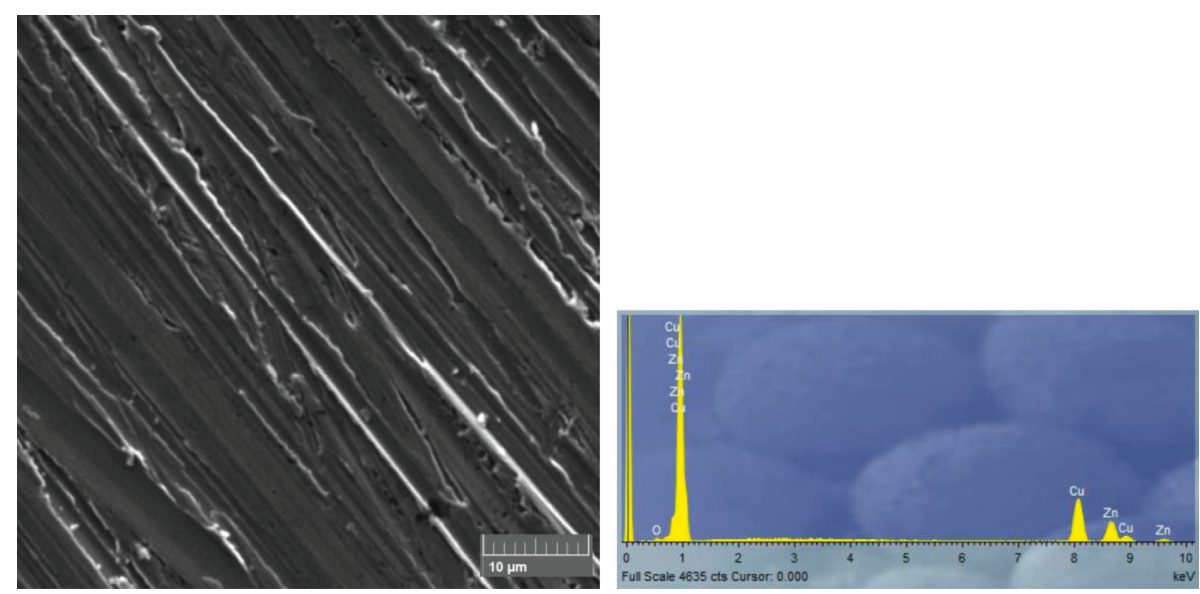

(a)
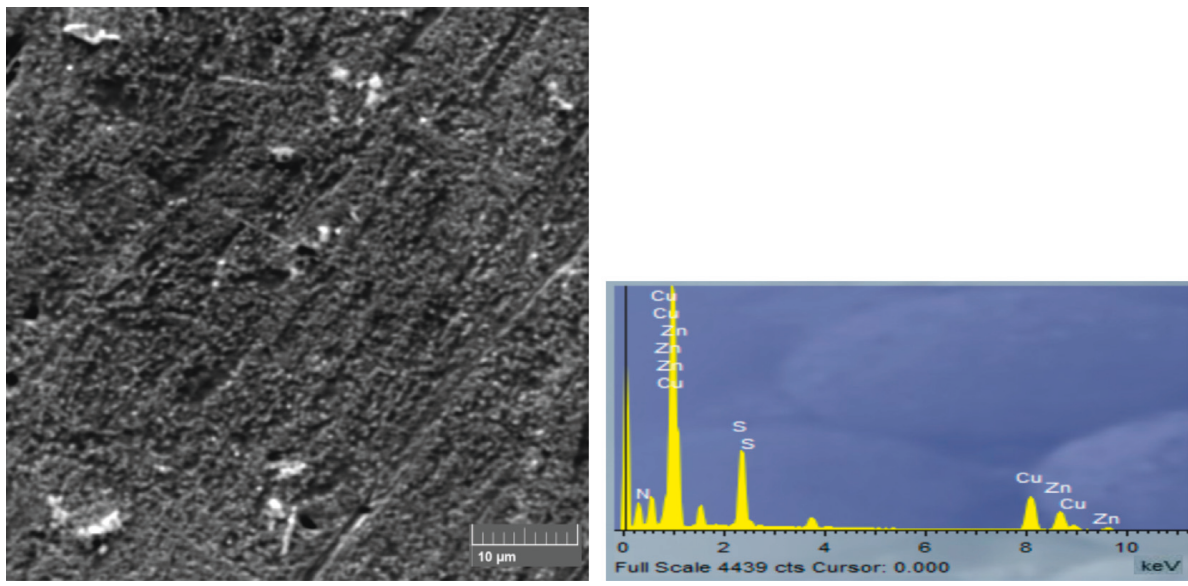

(b)
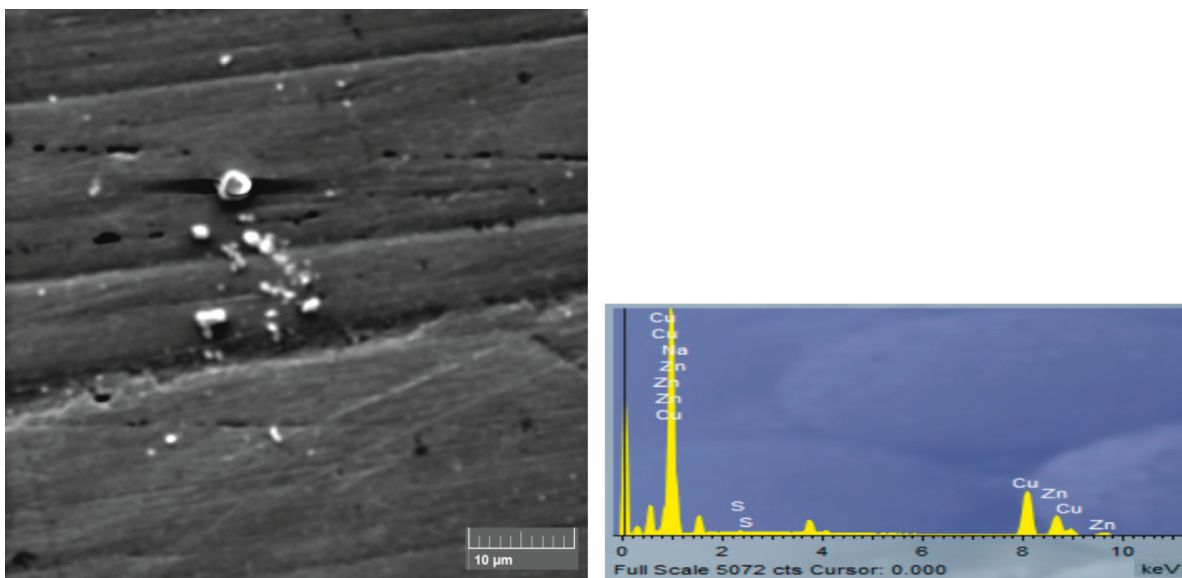

(c)

FIGURE 9: SEM and EDS analysis of (a) the polished brass surface without any treatment, (b) the brass surface after immersion of the electrode for $1140 \mathrm{~min}$ in $1 \cdot 10^{-2} \mathrm{M}$ cysteine solution, and (c) the brass surface after immersion of the electrode for $4080 \mathrm{~min}$ in $1 \cdot 10^{-3} \mathrm{M}$ SDS solution.

were calculated on the basis of corrosion parameters, and it was noticed that $R_{\mathrm{p}}$ values increase when immersion period of brass electrode in cysteine and SDS solutions increases. Moreover, increase of polarization resistance values points to adsorption of cysteine and SDS molecules on the electrode surface. $R_{\mathrm{p}}$ and IE values presented in Tables 2 and 4 indicate that increase of $R_{\mathrm{p}}$ values is accompanied by the increase of IE values. Accordingly, inhibitory effect as well as inhibition efficiency of cysteine depends on adsorption of inhibitor molecules on the electrode surface. 


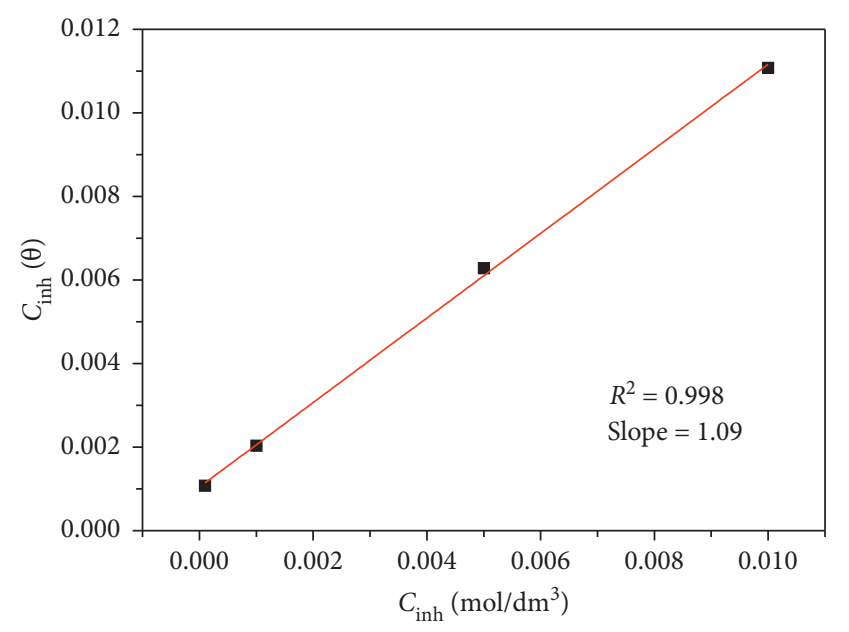

FIGURE 10: Langmuir adsorption isotherm of cysteine on the brass surface.

3.5. Surface Analysis. Figure 9 shows SEM-EDS images of polished brass surface without any treatment and after the immersion of the electrode for 1140 and $4080 \mathrm{~min}$ in $1 \cdot 10^{-2} \mathrm{M}$ cysteine and $1 \cdot 10^{-3} \mathrm{M}$ SDS solutions, respectively.

According to Figure 9, it can be seen that after pretreatment in cysteine and SDS solutions, the electrode surface is changed. Moreover, on the brass surface, which is present in Figures 9(b) and 9(c), exist adsorbed species that are not found on the clean brass surface (Figure 9(a)). EDS results show that sulfur and nitrogen are present on the brass surface after pretreatment in cysteine solution. Nonetheless, sodium and sulfur were detected on the electrode surface after brass pretreatment in SDS solution. These atoms derive from cysteine and SDS molecules, and their presence indicates that adsorption of mentioned compounds occurs on active sites on the brass surface.

3.6. Adsorption Isotherms. The adsorption of the inhibitor on the metal surface is one of the most important stages in the inhibition mechanism. The inhibitory effect may be associated with physical adsorption or with chemisorption of the tested inhibitor $[11,30]$. During the physical adsorption process, the metal surface must be electrically charged. Also, charged species must be present in the solution. Besides, presence of vacant low-energy electron orbital in metal structure is necessary for chemisorption process. Furthermore, inhibitors with relatively weakly bonded electrons or heteroatoms with lone pair electrons in molecules are necessary for chemical adsorption [57].

Moreover, useful information on the adsorption mechanism can be obtained from adsorption isotherms. Mechanism of adsorption of cysteine on the brass surface was examined using a number of different adsorption isotherms: Langmuir, Freundlich, Temkin, and Flory-Huggins.

According to correlation coefficient $\left(R^{2}\right)$ for tested isotherms, the Langmuir adsorption isotherm best describes the process of adsorption of cysteine on the electrode surface (Figure 10).
Values of the slope calculated according to the Langmuir adsorption isotherm indicate that, at one active site on the electrode surface, one molecule of inhibitor is adsorbed. Adsorption of cysteine in hydrochloric acid solution can be regarded as substitution process between the inhibitor in the aqueous phase and water molecules on the brass surface [58]:

$$
\mathrm{Inh}_{(\text {sol })}+\mathrm{H}_{2} \mathrm{O}_{(\mathrm{ads})}=\mathrm{Inh}_{(\mathrm{ads})}+\mathrm{H}_{2} \mathrm{O}_{(\text {sol })}
$$

Adsorption energy is calculated using the following equation:

$$
-\Delta G=\left[\ln K-\ln \left(\frac{1}{55.5}\right)\right] \cdot R T,
$$

where $K$ is the constant of the adsorption process ( $\left.\mathrm{dm}^{3} / \mathrm{mol}\right), \Delta G$ is the adsorption energy ( $\left./ / \mathrm{mol}\right), R$ is the universal gas constant $(\mathrm{J} / \mathrm{K} \cdot \mathrm{mol})$, and $T$ is the thermodynamic temperature $(295 \mathrm{~K})$.

Gibbs free energy of adsorption calculated according to the above equation has a value of $-31.5 \mathrm{~kJ} / \mathrm{mol}$ and indicates strong adsorption of cysteine on the electrode surface $[23,30]$.

\section{Conclusion}

Open circuit potential obtained in $\mathrm{HCl}$ solution with an addition of cysteine generally has more negative values in regard to OCP values obtained in bare $\mathrm{HCl}$ solution. This trend of shift of OCP values indicates adsorption of cysteine molecules on active sites on the electrode surface. Moreover, the shift of OCP values also points to the formation of protective complex in reaction between cysteine and copper oxidation products. Also, change of OCP values indicates that cysteine acts like cathodic type of inhibitor in acidic chloride solutions.

OCP values obtained in acidic chloride solutions have not significantly changed after electrode pretreatment in cysteine solution.

Nevertheless, OCP values reveal dominant adsorption of cysteine on anodic active sites on the electrode surface after brass modification in the SDS solution.

Cyclic voltammetry and linear potentiodynamic measurements performed in chloride solution with the addition of cysteine point to the formation of $\mathrm{Cu}(\mathrm{I})$-cysteine complex that significantly decreases the dissolution of brass. However, polarization curves obtained in $\mathrm{HCl}$ solution after electrode immersion in water solution of cysteine indicate weaker adsorption of cysteine molecules. Consequence of lower adsorption is formation of less stable protective film on the electrode surface. Nonetheless, potentiodynamic measurements achieved in acidic solution after pretreatment in SDS solution indicate charge change on the electrode surface. This leads to the facilitated adsorption of cysteine on anodic active sites and promotes the influence of inhibitor on decrease of anodic corrosion processes. Nonetheless, inhibition efficiency of cysteine is the highest after electrode pretreatment in SDS solution.

SEM-EDS investigation indicates that the changes in the morphology of the electrode surface after treatment in cysteine and SDS solutions occur which is caused by 
adsorption of cysteine and SDS molecules on the active sites on the brass surface.

Adsorption of the cysteine on the brass surface in $0.05 \mathrm{M}$ $\mathrm{HCl}$ solution obeys the Langmuir adsorption isotherm.

\section{Data Availability}

We have no publicly archived datasets so data is not publicly available but data can be provided on request.

\section{Conflicts of Interest}

The authors declare that they have no conflicts of interest.

\section{Acknowledgments}

The authors gratefully acknowledge the financial support of the Ministry of Education, Science and Technological Development of Republic of Serbia through the Project no. 172031.

\section{References}

[1] K. Ismail, "Evaluation of cysteine as environmentally friendly corrosion inhibitor for copper in neutral and acidic chloride solutions," Electrochimica Acta, vol. 52, no. 28, pp. 7811-7819, 2007.

[2] S. Zaferani, M. Sharifi, D. Zaarei, and M. Shishesaz, "Application of eco-friendly products as corrosion inhibitors for metals in acid pickling processes-A review," Journal of Environmental Chemical Engineering, vol. 1, no. 4, pp. 652657, 2013.

[3] G. Abd El-Hafez and W. Badawy, "The use of cysteine, $\mathrm{N}$-acetyl cysteine and methionine as solutions, environmentally friendly corrosion inhibitors for $\mathrm{Cu}-10 \mathrm{Al}-5 \mathrm{Ni}$ alloy in neutral chloride," Electrochimica Acta, vol. 108, pp. 860866, 2013.

[4] E. S. M. Sherif, R. M. Erasmus, and J. D. Comins, "Inhibition of copper corrosion in acidic chloride pickling solutions by 5 (3-aminophenyl)-tetrazole as a corrosion inhibitor," Corrosion Science, vol. 50, no. 12, pp. 3439-3445, 2008.

[5] E. S. M. Sherif and S. M. Park, "Inhibition of copper corrosion in $3.0 \% \mathrm{NaCl}$ solution by $\mathrm{N}$-phenyl-1,4-phenylenediamine corrosion, passivation, and anodic films," Journal of the Electrochemical Society, vol. 152, no. 10, pp. B428-B433, 2005.

[6] M. M. Antonijevic, S. C. Alagic, M. B. Petrovic, M. B. Radovanovic, and A. T. Stamenkovic, "The influence of $\mathrm{pH}$ on electrochemical behavior of copper in presence of chloride ions," International Journal of Electrochemical Science, vol. 4, pp. 516-524, 2009.

[7] M. M. Antonijevic, S. M. Milic, M. D. Dimitrijevic, M. B. Petrovic, M. B. Radovanovic, and A. T. Stamenkovic, “The influence of $\mathrm{pH}$ and chlorides on electrochemical behavior of copper in the presence of benzotriazole," International Journal of Electrochemical Science, vol. 4, pp. 962-979, 2009.

[8] S. M. Milic and M. M. Antonijevic, "Some aspects of copper corrosion in presence of benzotriazole and chloride ions," Corrosion Science, vol. 51, no. 1, pp. 28-34, 2009.

[9] M. M. Antonijevic, S. M. Milic, S. M. Serbula, and G. D. Bogdanovic, "The influence of chloride ions and benzotriazole on the corrosion behavior of Cu37Zn brass in alkaline medium," Electrochimica Acta, vol. 50, no. 18, pp. 3693-3701, 2005.
[10] M. M. Antonijevic, S. M. Milic, M. B. Radovanovic, M. B. Petrovic, and A. T. Stamenkovic, "Influence of $\mathrm{pH}$ and chlorides on electrochemical behavior of brass in presence of benzotriazole," International Journal of Electrochemical Science, vol. 4, pp. 1719-1734, 2009.

[11] M. B. Radovanović, M. B. Petrović, A. T. Simonović, S. M. Milić, and M. M. Antonijević, "Cysteine as a green corrosion inhibitor for Cu37Zn brass in neutral and weakly alkaline sulphate solutions," Environmental Science and Pollution Research, vol. 20, no. 7, pp. 4370-4381, 2013.

[12] K. Ramji, D. R. Cairns, and S. Rajeswari, "Synergistic inhibition effect of 2-mercaptobenzothiazole and Tween-80 on the corrosion of brass in $\mathrm{NaCl}$ solution," Applied Surface Science, vol. 254, no. 15, pp. 4483-4493, 2008.

[13] D. Q. Zhang, Q. R. Cai, X. M. He, L. X. Gao, and G. D. Zhou, "Inhibition effect of some amino acids on copper corrosion in $\mathrm{HCl}$ solution," Materials Chemistry and Physics, vol. 112, no. 2, pp. 353-358, 2008.

[14] O. Olivares, N. V. Likhanova, B. Gomez et al., "Electrochemical and XPS studies of decylamides of a-amino acids adsorption on carbon steel in acidic environment," Applied Surface Science, vol. 252, no. 8, pp. 2894-2909, 2006.

[15] W. A. Badawy, K. M. Ismail, and A. M. Fathi, "Corrosion control of $\mathrm{Cu}-\mathrm{Ni}$ alloys in neutral chloride solutions by amino acids," Electrochimica Acta, vol. 51, no. 2, pp. 4182-4189, 2006.

[16] H. Ashassi-Sorkhabi, Z. Ghasemi, and D. Seifzadeh, "The inhibition effect of some amino acids towards the corrosion of aluminum in $1 \mathrm{M} \mathrm{HCl}+1 \mathrm{M} \mathrm{H}_{2} \mathrm{SO}_{4}$ solution," Applied Surface Science, vol. 249, no. 1-4, pp. 408-418, 2005.

[17] A. T. Simonović, M. B. Petrović, M. B. Radovanović, S. M. Milić, and M. M. Antonijević, "Inhibition of copper corrosion in acidic sulphate media by eco-friendly amino acid compound," Chemical Papers, vol. 68, no. 3, pp. 362-371, 2014.

[18] G. Gece and S. Bilgiç, "A theoretical study on the inhibition efficiencies of some amino acids as corrosion inhibitors of nickel," Corrosion Science, vol. 52, no. 10, pp. 3435-3443, 2010.

[19] M. B. Petrović, A. T. Simonović, M. B. Radovanović, S. M. Milić, and M. M. Antonijević, "Influence of purine on copper behavior in neutral and alkaline sulfate solutions," Chemical Papers, vol. 66, no. 7, pp. 664-676, 2012.

[20] M. Scendo, "The effect of purine on the corrosion of copper in chloride solutions," Corrosion Science, vol. 49, no. 2, pp. 373-390, 2007.

[21] M. Scendo, "Corrosion inhibition of copper by purine or adenine in sulphate solutions," Corrosion Science, vol. 49, no. 10, pp. 3953-3968, 2007.

[22] M. Levin, P. Wiklund, and C. Leygraf, "Bioorganic compounds as copper corrosion inhibitors in hydrocarbon media," Corrosion Science, vol. 58, pp. 104-114, 2012.

[23] M. B. Radovanović, A. T. Simonović, M. B. Petrović, S. M. Milić, and M. M. Antonijević, "Influence of purine on brass behavior in neutral and alkaline sulphate solutions," International Journal of Electrochemical Science, vol. 7, pp. 11796-11810, 2012.

[24] M. M. Antonijevic, S. M. Milic, and M. B. Petrovic, "Films formed on copper surface in chloride media in the presence of azoles," Corrosion Science, vol. 51, no. 6, pp. 1228-1237, 2009.

[25] K. F. Khaled, "Experimental and atomistic simulation studies of corrosion inhibition of copper by a new benzotriazole derivative in acid medium," Electrochimica Acta, vol. 54, no. 18, pp. 4345-4352, 2009.

[26] H. Otmaĉič, J. Telegdi, K. Papp, and E. S. Lisac, "Protective properties of an inhibitor layer formed on copper in neutral 
chloride solution," Journal of Applied Electrochemistry, vol. 34, no. 5, pp. 545-550, 2004.

[27] E. S. M. Sherif, A. M. El-Shamy, M. M. Ramla, and A. O. H. ElNazhaw, "5-(Phenyl)-4H-1,2,4-triazole-3-thiol as a corrosion inhibitor for copper in $3.5 \% \mathrm{NaCl}$ solutions," Materials Chemistry and Physics, vol. 102, no. 2-3, pp. 231-239, 2007.

[28] E. M. Sherif and S. M. Park, "2-Amino-5-ethyl-1,3,4-thiadiazole as a corrosion inhibitor for copper in $3.0 \% \mathrm{NaCl}$ solutions," Corrosion Science, vol. 48, no. 12, pp. 4065-4079, 2006.

[29] M. Cubillos, M. Sancy, J. Pavez et al., "Influence of 8-aminoquinoline on the corrosion behaviour of copper in $0.1 \mathrm{M} \mathrm{NaCl}$," Electrochimica Acta, vol. 55, no. 8, pp. 2782-2792, 2010.

[30] M. B. Petrović, M. B. Radovanović, A. T. Simonović, S. M. Milić, and M. M. Antonijević, "The effect of cysteine on the behaviour of copper in neutral and alkaline sulphate solutions," International Journal of Electrochemical Science, vol. 7, pp. 9043-9057, 2012.

[31] H. Saifi, M. C. Bernard, S. Joiret, K. Rahmouni, H. Takenouti, and B. Talhi, "Corrosion inhibitive action of cysteine on $\mathrm{Cu}-30 \mathrm{Ni}$ alloy in aerated $0.5 \mathrm{M} \mathrm{H}_{2} \mathrm{SO}_{4}$," Materials Chemistry and Physics, vol. 120, no. 2-3, pp. 661-669, 2010.

[32] A. V. Polunin, A. P. Pchelnikov, V. V. Losev, and I. K. Marshakov, "Electrochemical studies of the kinetics and mechanism of brass dezincification," Electrochimica Acta, vol. 27, no. 4, pp. 467-475, 1982.

[33] H. Fan, S. Li, Z. Zhao, H. Wang, Z. Shi, and L. Zhang, "Inhibition of brass corrosion in sodium chloride solutions by self-assembled silane films," Corrosion Science, vol. 53, no. 12, pp. 4273-4281, 2011.

[34] A. M. Shams El Din, M. E. E. Dahshan, and A. M. Taj El Din, "Dissolution of copper and copper-nickel alloys in aerated dilute HC1 solutions," Desalination, vol. 130, no. 1, pp. 89-97, 2000.

[35] D. Q. Zhang, Q. R. Cai, L. X. Gao, and K. Y. Lee, "Effect of serine, threonine and glutamic acid on the corrosion of copper in aerated hydrochloric acid solution," Corrosion Science, vol. 50, no. 12, pp. 3615-3621, 2008.

[36] E. M. Sherif and S. M. Park, "Effect of 2-amino-5-ethylthio1,3,4-thiadiazole on copper corrosion as a corrosion inhibitor in aerated acidic pickling solutions," Electrochimica Acta, vol. 51, no. 28, pp. 6556-6562, 2006.

[37] D. Q. Zhang, L. X. Gao, Q. R. Cai, and K. Y. Lee, "Inhibition of copper corrosion by modifying cysteine self-assembled film with alkylamine/alkylacid compounds," Materials and Corrosion, vol. 61, no. 1, pp. 16-21, 2010.

[38] D. Li, S. Chen, S. Zhao, and H. Ma, "The corrosion inhibition of the self-assembled $\mathrm{Au}$, and Ag nanoparticles films on the surface of copper," Colloids and Surfaces A: Physicochemical and Engineering Aspects, vol. 273, no. 1-3, pp. 16-23, 2006.

[39] J. P. Ferreira, J. A. Rodrigues, and I. T. Elias de Fonseca, "Copper corrosion in buffered and non-buffered synthetic seawater: a comparative study," Journal of Solid State Electrochemistry, vol. 8, no. 4, pp. 260-271, 2004.

[40] J. B. Matos, L. P. Pereira, S. M. L. Agostinho, O. E. Barcia, G. G. O. Cordeiro, and E. D'Elia, "Effect of cysteine on the anodic dissolution of copper in sulfuric acid medium," Journal of Electroanalytical Chemistry, vol. 570, no. 1, pp. 91-94, 2004.

[41] A. Rigo, A. Corazza, M. L. Paolo, M. Rossetto, R. Ugolini, and M. Scarpa, "Interaction of copper with cysteine: stability of cuprous complexes and catalytic role of cupric ions in anaerobic thiol oxidation," Journal of Inorganic Biochemistry, vol. 98, no. 9, pp. 1495-1501, 2004.

[42] M. S. Deab, "Interaction of cysteine and copper ions on the surface of iron: EIS, polarization and XPS study," Materials Chemistry and Physics, vol. 129, no. 1-2, pp. 223-227, 2011.
[43] Z. Dursun and G. Nişli, "Voltammetric behavior of copper(I) oxide modified carbon paste electrode in the presence of cysteine and ascorbic acid," Talanta, vol. 63, no. 4, pp. 873-878, 2004.

[44] L. Pecci, G. Montefoschi, G. Musci, and D. Cavallini, "Novel findings on the copper catalysed oxidation of cysteine," Amino Acids, vol. 13, no. 3-4, pp. 355-367, 1997.

[45] E. A. Tumanova, A. Yu Safronov, and A. V. Kapustin, "Mechanism of electrochemical interaction between sulfurcontaining amino acids and anodically polarized platinum electrode," Russian Journal of Electrochemistry, vol. 37, no. 9, pp. 972-975, 2001.

[46] R. Tremont, H. D. J. Cardona, J. G. Orozco, R. J. Castro, and C. R. Cabrera, "3-Mercaptopropyltrimethoxysilane as a $\mathrm{Cu}$ corrosion inhibitor in $\mathrm{KCl}$ solution," Journal of Applied Electrochemistry, vol. 30, no. 6, pp. 737-743, 2000.

[47] M. A. Migahed and I. F. Nassar, "Corrosion inhibition of Tubing steel during acidization of oil and gas wells," Electrochimica Acta, vol. 53, no. 6, pp. 2877-2882, 2008.

[48] A. R. H. Zadeh, I. Danaee, and M. H. Maddahy, "Thermodynamic and adsorption behavior of medicinal nitramine as a corrosion inhibitor for AISI steel alloy in $\mathrm{HCl}$ solution," Journal of Materials Science and Technology, vol. 29, no. 9, pp. 884-892, 2013.

[49] O. Ghasemi, I. Danaee, G. R. Rashed, M. Rashvandavei, and M. H. Maddahy, "The inhibition effect of synthesized 4hydroxybenzaldehyde-1,3-propandiamine on the corrosion of mild steel in $1 \mathrm{M} \mathrm{HCl,"} \mathrm{Journal} \mathrm{of} \mathrm{Materials} \mathrm{Engineering} \mathrm{and}$ Performance, vol. 22, no. 4, pp. 1054-1063, 2013.

[50] G. Bereket and A. Yurt, "The inhibition effect of amino acids and hydroxyl carboxylic acids on pitting corrosion of aluminium alloy 7075," Corrosion Science, vol. 43, no. 6, pp. 1179-1195, 2001.

[51] A. Popova, E. Sokolova, S. Raicheva, and M. Christov, "AC and DC study of the temperature effect on mild steel corrosion in acid media in the presence of benzimidazole derivatives," Corrosion Science, vol. 45, no. 1, pp. 33-58, 2003.

[52] A. Lalitha, S. Ramesh, and S. Rajeswari, "Surface protection of copper in acid medium by azoles and surfactants," Electrochimica Acta, vol. 51, no. 1, pp. 47-55, 2005.

[53] T. Zhao and G. Mu, "The adsorption and corrosion inhibition of anion surfactants on aluminium surface in hydrochloric acid," Corrosion Science, vol. 41, no. 10, pp. 1937-1944, 1999.

[54] R. F. V. Villamil, P. Corio, J. C. Rubim, and S. M. L. Agostinho, "Sodium dodecylsulfate/benzotriazole synergistic effect as an inhibitor of processes on copper chloridric acid interfaces," Journal of Electroanalytical Chemistry, vol. 535, no. 1-2, pp. 75-83, 2002.

[55] H. Ma, S. Chen, B. Yin, S. Zhao, and X. Liu, "Impedance spectroscopic study of corrosion inhibition of copper by surfactants in the acidic solutions," Corrosion Science, vol. 45, no. 5, pp. 867-882, 2003.

[56] M. B. Radovanovic and M. M. Antonijevic, "Inhibition of brass corrosion by 2-mercapto-1-methylimidazole in weakly alkaline solution," Journal of Materials Engineering and Performance, vol. 25, no. 3, pp. 921-937, 2016.

[57] M. Scendo and J. Trela, "Inhibition effect of N-(2chlorobenzylidene)-4-acetylaniline on the corrosion of stainless steel," International Journal of Electrochemical Science, vol. 8, pp. 11951-11971, 2013.

[58] W. Durnie, R. De Marco, A. Jefferson, and B. Kinsella, "Development of a structure-activity relationship for oil field corrosion inhibitors," Journal of the Electrochemical Society, vol. 146, no. 5, pp. 1751-1756, 1999. 


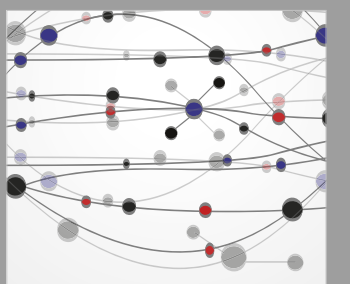

The Scientific World Journal
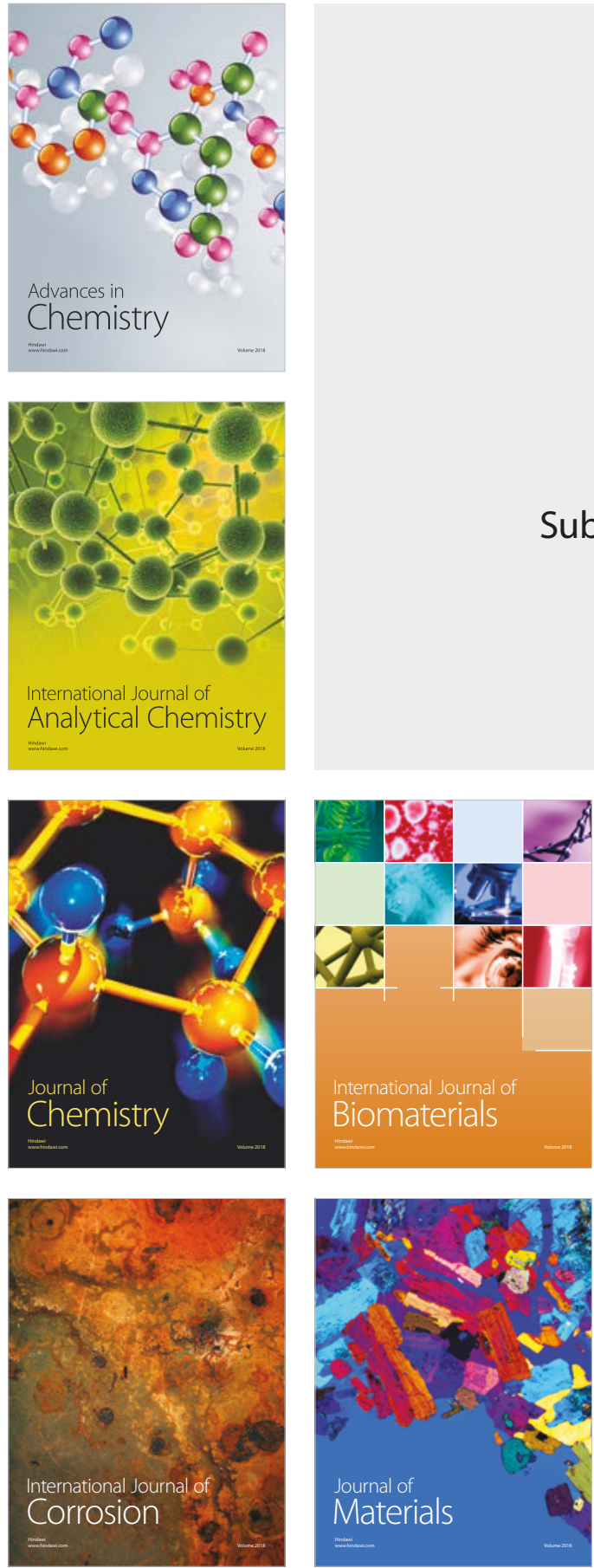

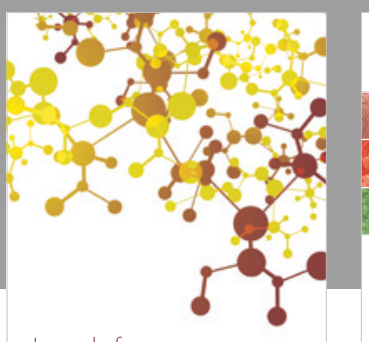

Journal of

Applied Chemistry
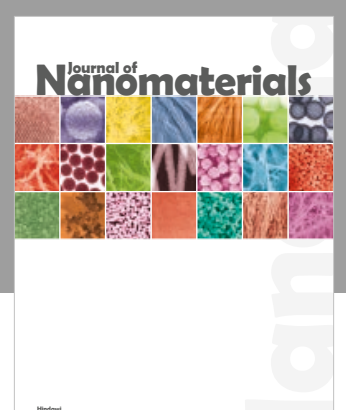

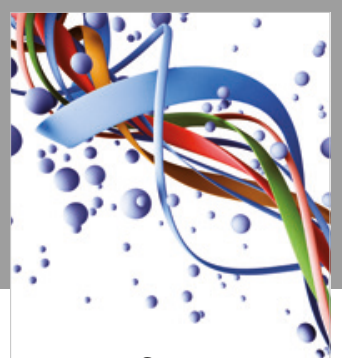

Scientifica

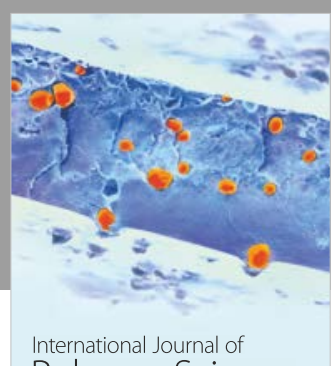

Polymer Science

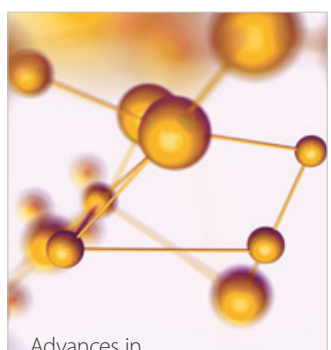

Physical Chemistry
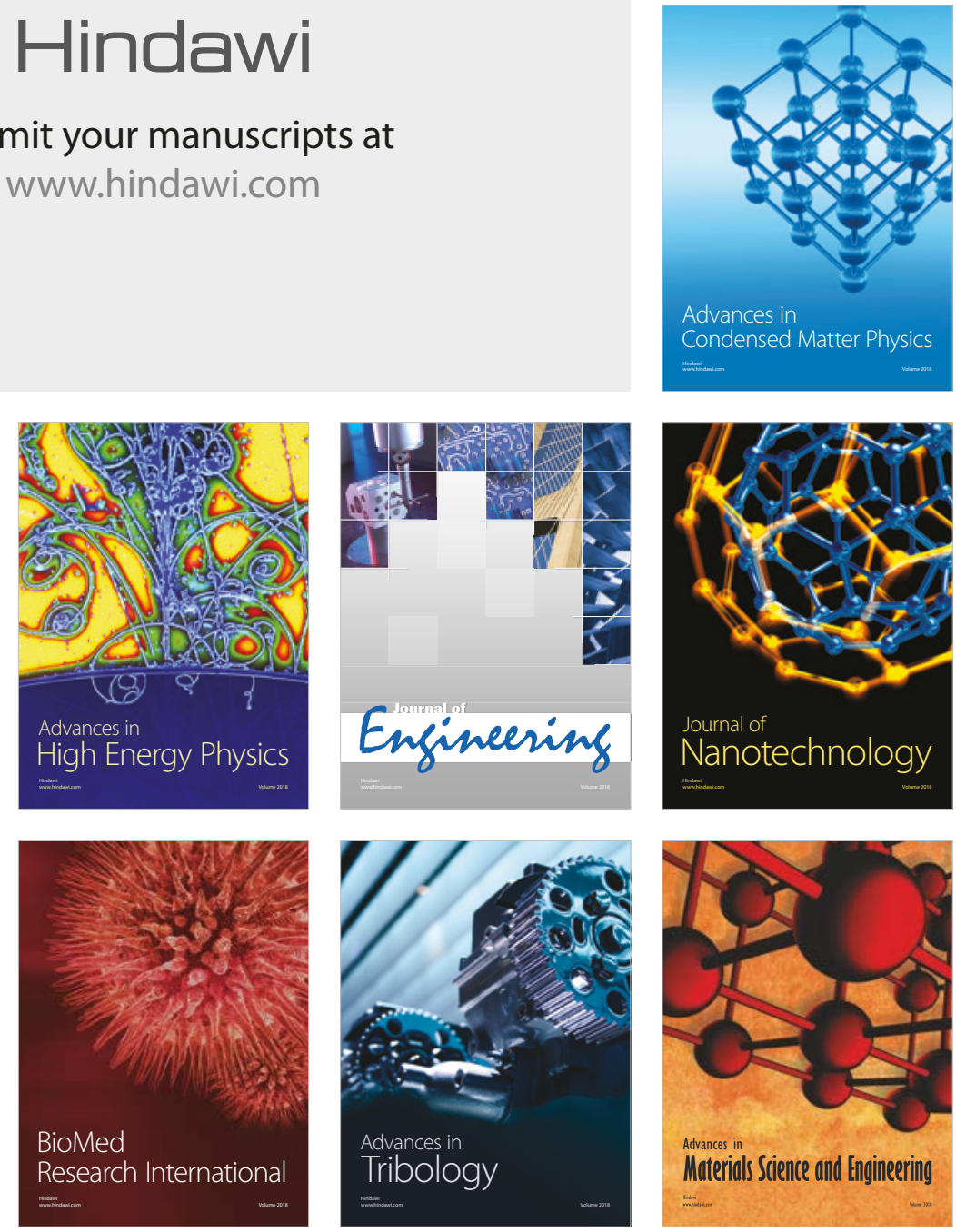